\section{Cahiers de Narratologie}

Analyse et théorie narratives

$20 \mid 2011$

Voix off et narration cinématographique

\title{
La transmission narrative
}

\section{Fausto Boni}

\section{OpenEdition}

\section{Journals}

Édition électronique

URL : http://journals.openedition.org/narratologie/6372

DOI : 10.4000/narratologie.6372

ISSN : 1765-307X

Éditeur

LIRCES

\section{Référence électronique}

Fausto Boni, «La transmission narrative », Cahiers de Narratologie [En ligne], 20 | 2011, mis en ligne le 18 juillet 2011, consulté le 19 avril 2019. URL : http://journals.openedition.org/narratologie/6372 ; DOI : $10.4000 /$ narratologie. 6372

Ce document a été généré automatiquement le 19 avril 2019

Article L.111-1 du Code de la propriété intellectuelle. 


\section{La transmission narrative}

\section{Fausto Boni}

1 Transmettre, au sens d'envoyer un texte au-delà d'un contexte donné de nature individuelle, locale, ou temporelle, paraîtrait être l'une des fonctions de la littérature en tant que forme d'expression humaine. L'idée selon laquelle celui ou celle qui écrit, parmi ses motivations, a l'intention de transmettre un texte qui, fondamentalement, prévoit la possibilité d'être compris par quelqu'un d'autre, dans un autre lieu ou un autre temps, semblerait une évidence. Naîtrait alors une relation entre l'auteur et le lecteur, qui serait fondée sur la transmission et sur l'hybridation.

De ce point de vue, la narration romanesque (étymologiquement narrer veut dire « faire connaître en racontant »), en tant que genre littéraire en prose, en tant que produit de l'acte de raconter, apparaîtrait à plus forte raison impliquée dans la transmission d'un contenu. Mais est-ce vraiment le cas? Le genre littéraire de la narration, dans sa forme actuellement la plus diffusée, celle du roman, est-il vraiment en mesure de transmettre un contenu? Et dans le cas où il serait en mesure de le faire, transmet-il de la connaissance, qu'elle soit conceptuelle ou autre?

3 Évoquer le problème de la transmission dans le cadre de la narration signifie engager préalablement deux niveaux d'approfondissement et ensuite vérifier, dans le genre narratif, si la répartition ultérieure entre high brow et low brow a des conséquences du point de vue de la transmission. Le premier cadre d'approfondissement que je souhaiterais développer concerne le thème de la légitimité de l'outil narratif pour transmettre du contenu.

\section{Mimésis et épistème}

4 La culture grecque archaïque a une conception mythique du langage. D'un côté elle identifie parole et être en faisant coïncider langage et réalité. D'un autre coté elle attribue aux actions et aux mots humains des "sphères de sens partiellement autonomes ${ }^{1}$ " affectées à des buts précis (oraisons judiciaires ou oraisons démonstratives, persuasives) et organisées hiérarchiquement (tragédie, comédie ; epos haut et epos bas); des régions de sens qui ne peuvent être reliées à un principe commun capable de les comprendre toutes. 
Le monde est pensé comme un agrégé d'événements individuels, hekasta, méthodiquement liés dans des figures généralisables, qui cependant ne deviennent pas des idées ou des lois immanentes à la réalité que dans la mesure où l'intervention technique les consolide et les réaffirme².

Platon, dans sa recherche des principes généraux pouvant donner des explications à propos de la nécessaire et universelle correspondance entre la réalité et les mots utilisés pour l'exprimer, ne se contente pas de la simple validité régionale des genres exprimée par la rhétorique et par la poétique anciennes, mais il veut opposer le logos philosophique - tout à la fois ratio et oratio - au mythos ancien - oratio sans ratio -. Il veut ainsi affirmer la capacité exclusive de la philosophie à comprendre la nature intelligible du réel, à travers l'utilisation «correcte » de l'argumentation rationnelle. Dans l'intention platonicienne, l'aptitude exclusive du discours philosophique de contempler et communiquer les essences (ou idées) rend subalternes tous les autres discours qui s'occupent, au contraire, de cas individuels et de choses singulières (par définition muables et contradictoires).

6 La révolution épistémologique platonicienne consiste exactement dans l'affirmation que seules les idées, contemplées par la connaissance rationnelle dans leur constance et universalité, peuvent être l'objet de véritable connaissance tandis qu'on ne peut avoir qu'une connaissance sensible, imparfaite et discutable des singularités situées dans le temps. Les choses singulières auxquelles, selon Platon, on accède par le moyen des sens, perdent de status ontologique par rapport aux idées. La relation que Platon établit entre objets sensibles et idées est donc comparable à celle qui existe entre apparence et réalité, ou, autrement dit, entre reproduction et modèle. Dans plusieurs passages de son œuvre, Platon se réfère à cette relation en utilisant la notion d'imitation, mimésis : les choses belles existent parce qu'elles imitent imparfaitement l'idée du beau ${ }^{3}$. De cette relation entre idées et monde sensible descend directement l'argument théorétique que Platon met au point dans la République, selon lequel les pratiques mimétiques des artistes, qui à travers leurs ouvres fixent des êtres ou des événements particuliers et visibles en les décrivant, resteraient à la surface sensible et donc apparente de la réalité. Cette superficialité produit deux conséquences que Platon dénonce afin de substituer, dans la paideia grecque, l'hégémonie des philosophes sur celle des artistes en général et des poètes en particulier. D'une part, les imitateurs n'auraient pas la capacité de saisir les essences universelles abstraites dont seule la connaissance, patrimoine du nouveau discours philosophique, pourrait permettre la véritable épistème. Adhérant inconsciemment à une ontologie du "réalisme naïf ", ils seraient en mesure de ne produire que de blafardes imitations linguistiques des singularités sensibles qui, imparfaites par elles-mêmes, ne sont que des copies des modèles idéaux. D'autre part, la transmission de ces contenus, aussi évanescents qu'inessentiels, favorise la communication de contenus latents, émotionnels plutôt que rationnels, qui imposerait à la personne jouissant de l'œuvre, l'acceptation acritique d'une interprétation partielle de la réalité.

7 À partir de la Poétique, Aristote, suivant l'optique de Platon, trace les limites de la mimésis littéraire à travers la distinction des moyens utilisés afin d'imiter la réalité. La mimésis qui imite la réalité en utilisant les mots, c'est-à-dire la littérature, est définie poiesis et a ses aspects fondamentaux : une histoire à raconter et une contingence de l'événement ; une forme déterminée, un ordre rationnel, et donc humain, au moyen duquel on présente les faits/objets du récit. Deux éléments au moins de la taxinomie aristotélicienne survivent encore : l'idée que « l'intrigue n'est pas une copie mais une interprétation du monde » et, 
par conséquent, l'idée qu'une interprétation du monde, pour se concrétiser, a besoin de construire un "système de faits" rationnel, donc tout à fait hors de nature, qui fait référence à l'ontologie réaliste, dont on a déjà indiqué les limites. Les conditions de ce qui est possible pour pouvoir développer une intrigue, à savoir les "structures primaires " sur lesquelles se fonde l'ontologie, sont les suivantes : temps et espace, permettant de faire évoluer les personnages horizontalement et verticalement; un décalage émotif qui permet de faire bouger les personnages à l'intérieur du récit; un cadre physique et culturel qui permet de fournir une épaisseur existentielle au mouvement des personnages ; un système social qui permet de circonscrire le mouvement d'ensemble des personnages.

8 Dès le début de la réflexion occidentale, seule la mimésis, grâce à sa représentation du hasard, peut sauver la mémoire des choses, des gestes, des mots, des passions, des pensées, qui se sont passés dans le temps. Jusqu'à une époque très récente, la connaissance du monde envisageait un clivage net entre mimésis et épistème et soulignait la capacité de l'idée à réduire le temps à la pensée et à unifier les particuliers aux universels.

\section{Mimésis et romantisme}

C'est le romantisme qui, à travers la réévaluation de l'art comme forme de connaissance tout aussi digne que la pensée, renouvelle la relation mimétique, donc narrative, avec le monde : l'essentiel n'est plus quelque chose de fantomatique à découvrir en soi, mais se développe dans la réalité habitée par des êtres finis et leurs formes de vie.

Le changement des rapports de force édictés par Platon se manifeste dans le domaine narratif avec le roman nouveau de Balzac. Dans l'avant-propos de La Comédie humaine, Balzac affirme que le romancier du XIX ${ }^{\mathrm{e}}$ siècle est le véritable expert de l'espèce humaine, le seul à avoir la légitimité d'écrire l'interprétation de l'« immense assemblage de figures, de passions et d'événements ${ }^{4} »$ dont est faite la société. C'est le seul qui, en plus de sa capacité à représenter toute la variété de la vie à travers l'enchevêtrement des passions, des désirs et des actions qui définissent l'existence d'êtres singuliers représentés dans leur cadre de vie, est également capable de mettre en évidence les lois, les universaux qui gouvernent les changements de la société. La littérature est d'ailleurs un art mimétique, elle opère par le biais des mots, mais en même temps possède un élément d'abstraction par rapport à l'expérience sensible, car le langage écrit est différent du langage parlé. À travers les mots, nous entrons dans les passions et nous accédons aux idées abstraites.

11 Si les essais philosophiques ou scientifiques se concentrent sur les classes de phénomènes et sur les lois qu'on peut en tirer, le roman met au centre de sa construction l'immersion mimétique dans des histoires singulières, à partir desquelles on accède au domaine de l'abstrait, mais qui restent toujours le point de départ du développement romanesque.

Comme l'affirme Sergio Givone, l'histoire universelle en forme de philosophie s'attache, jusqu'à Hegel (qui dans la Phénoménologie raconte « la seule histoire vraie, la seule histoire réelle ») à fonder la raison occidentale sans que personne ne reconnaisse aucune légitimité de connaissance ni au récit en tant que genre ni au roman en particulier. 


\section{Le roman de l'Esprit}

13 Avant Hegel c'est Leibniz qui imagine "le roman de l'histoire universelle » : la seule histoire qui se nie d'elle-même en se faisant histoire véritable. Pour ne pas se substituer à Dieu (le seul en mesure d'écrire ce roman-là), Leibniz se garde bien de faire ce que Hegel fait, c'est-à-dire : écrire le livre «qui raconte l'histoire du monde telle qu'elle se passe véritablement ${ }^{5}$ ». En écrivant la Phénoménologie de l'esprit, Hegel s'installe "dans la bibliothèque qui est l'esprit de Dieu ${ }^{6} »$ et écrit la seule histoire véritable du point de vue de l'Idée, « le dernier mot sur le monde ${ }^{7}$. I Il raconte l'histoire de la conscience qui, grâce à un processus d'évolution qui se déroule en passant par des étapes bien précises, s'empare du monde extérieur et se révèle enfin à elle-même en tant qu'Idée, Esprit absolu. Cette évolution confirme l'existence d'une vérité atemporelle et métaphysique, dans laquelle le monde extérieur est à la fin «absorbé ». Le concept, c'est-à-dire l'Idée, la conscience, passe à travers le temps mais il ne s'y dissout pas, car il se sert du temps pour arriver à se découvrir tel qu'il est. « Du moment que l'histoire est racontée à la lumière du concept, c'est le concept qui gagne sur l'histoire ${ }^{8}$ ".

Voilà encore deux mondes en opposition. D'une part, le monde de la vie comme représentation surgissant d'une conscience qui est en mesure d'atteindre uniquement ce que Platon aurait nommé la connaissance sensible. Une connaissance qui, dans la pluralité infinie des interprètes et des interprétables, ne pourrait que se perdre dans la variété inépuisable des perspectives possibles. De l'autre, le monde de l'Esprit, qui se redécouvre en tant que auto-conscience à la fin du parcours que la conscience fait dans le monde immanent. Les deux mondes, selon Hegel, trouvent une expression accomplie et une synthèse dans le roman en tant que projet de production de sens de la part d'un sujet autonome. Mais le dernier mot sur le monde de la vie, la vérité essentielle, c'est-à-dire l'accidentalité de ce monde par rapport au Moi absolu de l'Esprit, a déjà été dite par l'Esprit lui-même dans le roman unique qui est la Phénoménologie. Tous les autres romans, qui s'obstineraient à raconter le monde comme une « dimension infiniment ouverte » et une "région du possible», ne sont que des manifestations "de l'inactualité de l'art romantique ». Ils ne font que s'attarder à exprimer la "non-vérité », l'impossibilité de comprendre rationnellement un monde déjà compris dans la Phénoménologie.

La poiesis romanesque, dans la perspective hégélienne, risque de perdre la simple capacité mimétique que, de son côté, Platon reconnaissait aux poètes. Si, dans la perspective platonique, les phénomènes décrits par les poètes conservent une pâle trace de l'idée transcendante, au contraire, pour Hegel, la vérité romanesque oscille entre la futilité d'une fantaisie sans freins et la régression dans l'irréalité d'une réconciliation de l'Esprit jamais arrivée. Étant donné que l'Idée, l'unique et véritable réalité, s'aliène dans le monde pour arriver, à la fin du chemin de la connaissance, à se retrouver en soi-même, celui qui s'attarderait à raconter des histoires attribuerait un sens autonome aux événements du monde sensible, chose d'ailleurs impossible puisque le sens de ce monde se révèle seulement grâce à l'identification de l'intelligible et du sensible, qui se trouve au bout du chemin de l'Idée.

Dans la perspective hégélienne la poiesis romanesque essaye d'imiter platoniquement le monde, mais elle lui reste irrémédiablement étrangère. Elle est « ressemblante » parce qu'elle imite le monde en le reconstruisant au moyen d'une perspective partielle, qui 
cependant ne pourra jamais le saisir entièrement. Elle est irrémédiablement étrangère parce que, tout en imposant sa partialité, elle démontre la fausseté de la totalité.

Le roman donc, comme Hegel l'atteste par voie négative, se soustrait à la théorie. C'est la négation du regard entièrement déplié sur la réalité et capable de l'embrasser complètement jusqu'à en restituer l'image parfaite, le double [...]. Se soustrayant à la théorie, le roman lui tend un piège, la menace, en met en lumière le principe désagrégeant. Chaque roman est là pour démontrer que le regard total est illusion'.

\section{Mort de Dieu et fin de la métaphysique}

17 Le fait que, dans les siècles suivants aussi, le roman soit resté un moyen efficace pour former la «commune perception des événements ${ }^{10} »$, témoigne de l'incapacité du mouvement de la pensée à subordonner à soi-même la partialité dissolvante de la mimésis romanesque, laquelle tout au plus, dans sa permanence et son développement, dénonce sa nécessité irréductible.

Le discours né avec Nietzsche et devenu central après le changement poststructuraliste, relève la difficulté de faire coïncider être et pensée, à savoir la substantielle opacité de la pensée. Avec la « mort de Dieu » le monde devient tout à coup insensé et muet. L'Esprit hégélien n'arrive pas non plus à produire la totalité, à donner un sens au monde. La vérité, en tant que paradigme inaltérable, en tant que totalité auto-consciente et éternelle, en tant qu'identité toujours égale à elle-même, s'écroule, nous laissant déracinés et nomades. Le raisonnement abstrait ne fonctionne pas, ou plutôt, fonctionne comme toute autre forme : la pensée devient un " jeu linguistique ${ }^{11}$ » à côté d'autres jeux linguistiques. L'autorité de la métaphysique entre en crise faisant place à la mimésis et à la pensée abstraite. Le monde des essences s'effondre avec son corrélatif naturel : le monde des phénomènes. Le partage platonicien n'a plus raison d'être : «l'essentiel étant que la vérité est la puissance de la négation et de la contradiction qui agit sur le temps ${ }^{12}$. ». En paraphrasant le Crépuscule des idoles, on peut dire quece n'est pas le monde qui devient fable, c'est la fable qui peut être monde. La philosophie devient roman « et Zarathushtra peut enfin commercer à être raconté1 ${ }^{13}$. ».

19 C'est à travers Lukàcs que la théorie fait sa dernière tentative de réduire à soi-même la mimésis romanesque. Même en renversant le jugement de Hegel sur la valeur cognitive du roman, Lukàcs parviendra à identifier un point de vue absolu auquel ajuster le monde (qui cependant ne se situe pas à l'origine comme L'Esprit d'Hegel mais tout au plus à la fin en tant qu'histoire). Lukàcs ne veut pas résorber toute manifestation humaine dans une ontologie identitaire préétablie: l'époque de la suprématie de la métaphysique est révolue. Le roman n'est pas une forme d'art inactuelle, dépassée par la réflexion autoconsciente. Le roman est un point de vue sur le monde, un miroir du temps, « l'épopée du monde déserté par les dieux ${ }^{14}$.». En constatant la fin de la métaphysique, Lukàcs se rend compte que l'homme se trouve " jeté » dans un monde subitement étranger, inhospitalier et inhabité, mais il peut s'ingénier à le reconstruire, il peut essayer de lui donner un sens, puisque l'homme a toujours besoin d'un sens, d'une théorie, afin de reconstruire une maison commune. Ce sens ne pourra plus séparer les essences des apparences, il ne sera plus « objectif », mais, en tant que produit du nomadisme foncier de l'être humain, il sera problématique et subjectif. La forme de ce sens incertain est le roman en tant que mimésis littéraire de l'histoire des âmes qui s'aventurent dans le monde afin de se trouver. Le roman tend au même but que l'epos ancien, mais il ne peut jamais l'atteindre car, dans la 
société bourgeoise, les moyens pour atteindre des buts épiques sont hétérogènes et les résultats diamétralement opposés. Le roman est donc l'epos d'une société qui détruit la possibilité de la création épique ${ }^{15}$. D'après Lukàcs, le roman revendique son actualité, parce qu'il se place entre le monde qui a disparu (l'époque heureuse de l'histoire de l'humanité où l'épopée et la tragédie affirmaient l'unité organique de la vie et de son sens) et le monde à venir, dans lequel disparaîtra la division entre le public et le privé, entre l'individu et la société, entre la production sociale et l'appropriation privée. Par la représentation de l'action, la médiation narrative reconstruit la relation réelle entre l'homme et la société, entre l'homme et la nature. Elle ne reconstruit pas seulement la conscience qu'a l'homme de ces relations, mais l'être même, qui est le fondement de cette conscience. Seulement quand l'homme agit, par l'intermédiaire de l'être social, son essence véritable trouve son expression. L'imagination poétique, fantaisie poétique du narrateur, consiste à inventer un récit où cette essence de l'homme, cet élément typique de son être social, trouve son expression active. À travers le destin individuel ainsi figuré on peut voir les traits essentiels de l'être historique-concret, d'une forme sociale donnée ${ }^{16}$

Dans The Claim of Reason (Le droit de la raison), Stanley Cavell a noté la permanence d'une volonté hégémonique du discours philosophique par rapport à ce qu'il affirme être l'expression spécifique de l'homme et de son être au monde, à savoir l'écriture. À son avis, seule la littérature est en mesure de dialoguer avec l'humanisme. La philosophie s'acharne à construire des systèmes formels, même de type linguistique, qui comprennent le monde en l'objectivant. Une fois de plus, cela revient à s'éloigner du monde de la vie, à perdre de vue la communication intersubjective qui construit du sens à partir de perspectives particulières et limitées. Le langage, qui permet de conjuguer la description objective d'un fait et des valeurs, n'est pas le langage formel et abstrait, qui s'occupe des messages et qui construit des significations, mais le langage naturel et ordinaire, qui transmet des textes plongés dans des contextes, autrement dit : l'écriture. Selon Cavell, c'est dans l'écriture que « le sens est construit par la simulation ${ }^{17}$.».

L'idée de parler de la vérité comme d'une œuvre d'art et non comme d'une pure et simple découverte du résultat d'une recherche, découle de la conviction que toute œuvre, produite par l'esprit humain, ne reproduit pas exactement ce qui est, mais est chargée de subjectivité. Malgré une certaine résistance due à des "formations discursives ${ }^{18}$ " consolidées pendant deux millénaires, on peut dire qu'à partir de Nietzsche, l'idée « que l'image du monde transmise par les disciplines réflexives est une construction poïétique pas très différente de l'image du monde transmise par les arts ${ }^{19}$ » s'est renforcée.

Si les concepts doivent être créés, et les universaux construits, alors le philosophe ou le scientifique ne trouvent pas, mais inventent aussi le discours. Le scientifique devient un artiste et l'artiste accède au rang de scientifique : la mimésis fait de nouveau partie de ce que Foucault appelait les "jeux de vérité ». On assiste actuellement à une scission entre différents «jeux de vérité »: la mimésis et la pensée abstraite. Il s'agit d'un double mouvement qui, d'un côté, préserve la singularité individuelle en tant que valeur non contestable et, de l'autre, confirme toutes les limites du particularisme. Le triomphe parallèle de la forme narrative du roman et de la recherche scientifique remet en exergue la séparation irréductible entre deux conceptions de la réalité. Mimésis et pensée abstraite, comme nous l'avons dit, représentent deux jeux de vérité actuellement scindés, deux pôles difficiles à réunifier, qui résument la scission de l'homme moderne. Le roman, par sa capacité à représenter la particularité singulière et subjective des individus et en 
même temps l'universalité plurielle et objective du monde à laquelle ceux-ci sont assujettis, "est la forme de la totalité divisée »: il est à la fois "individualiste » et «systémique ${ }^{20} »$.

\section{Temps et espace}

Venons-en au problème des contenus que le récit serait en mesure de transmettre. De fait, deux formes de liens permettent à la mimésis narrative d'atteindre une nouvelle dignité. La première, la plus dépassée, est celle qui unit le temps et le récit. Ce lien, dès lors qu'il met en évidence la temporalité inhérente à la notion de vérité, autorise à privilégier le «monde de la vie» en opposition au «langage monde» métaphysique. La seconde forme de lien, la plus actuelle, unit l'espace et la narration. Ce lien, révélant l'aptitude du discours littéraire à interagir avec la perception et la production de l'espace, permet de redéfinir la fonction de la fiction. Grâce à ce travail d'interprétation, le récit n'est plus une reproduction mimétique du réel mais devient un lieu complexe et dynamique de construction de l'espace et de sa représentation.

À partir de la réflexion nietzschéenne, comme nous l'avons précisé, la question du rapport entre la vérité et le temps est de plus en plus problématique. Même si dans certains domaines de la philosophie et de la science on continue à juger la vérité comme quelque chose d'eternel qu'il s'agit seulement de découvrir, on assiste à la croissance de l'idée opposée, à savoir que la vérité, comme toutes les choses, est en devenir et qu'on peut lui redonner un sens, toujours partiel, seulement dans ses formes transitoires.

L'hypothèse de Ricœur, dans Temps et récit, consiste à montrer comment, en situant la vérité dans le temps, on la réduit, pour ainsi dire, à mesure d'homme et donc on ne la considère plus comme absolue mais toujours en transformation. Cela produit deux conséquences liées: le temps devient temps humain et, par conséquent, il est inévitablement décliné selon les paradigmes et les traits stylistiques du récit. Selon Ricœur, l'homme fait l'expérience du temps en racontant une histoire, c'est-à-dire qu'il l'expérimente en ordonnant les événements dans une succession. Mais raconter une histoire implique qu'on utilise le langage et cela complique les choses. Comme nous avons déjà vu, la métaphysique, saisissant le monde par le langage, s'est trouvée à manipuler un langage-monde plein de théorie mais sans vie. Dans la perspective de Ricœur, le langage n'est pas un monde, le monde est là ; le langage, dans une optique herméneutique, se réfère au monde en le préfigurant, en le configurant et en le figurant de nouveau. C'est-àdire que l'homme fait expérience du temps comme récit par moyen du langage qui imite le monde, lequel, de son côté, est toujours là. Le récit préfigure l'action à travers un système symbolique, configure un monde à travers une œuvre qui est de même un monde et, par conséquent, figure de nouveau l'expérience du temps du lecteur. Ce sont les mêmes opérations que chacun de nous accomplit quand il agit. Voilà alors de quelle façon le lecteur, à travers le récit, fait l'expérience d'un passé qui pourrait devenir futur, d'une pratique jamais faite qui pourrait devenir réelle.

26 La critique littéraire la plus récente a déplacé son attention de la représentation du temps à la représentation littéraire de l'espace. Elle a ainsi ouvert une nouvelle possibilité de transmission pour la mimésis narrative. Dans un horizon de discours qui désormais n'accepte plus une notion statique de vérité, la géocritique conçoit l'espace dynamiquement, soit comme le résultat de l'interaction de différents acteurs sociaux, soit comme conséquence du rapport entre espace intime et espace public. De même que 
l'approche herméneutique de Ricœur humanisait le temps et trouvait sa narrativité implicite, la perspective géocritique veut humaniser l'espace et voit la littérature comme un moyen actif capable de le produire. L'espace n'est pas simplement un fait, quelque chose de permanent qu'il suffit de décrire dans sa réalité (espace perçu). Il n'est pas un concept maîtrisable (espace connu), mais un espace vécu et donc imaginé, symbolisé. Le discours littéraire facilite le passage de l'espace réel à l'espace imaginaire, fonde l'espace, ou plutôt le co-fonde avec des autres disciplines. La géocritique ne place au centre de l'analyse ni l'œuvre ni l'auteur; elle veut placer au centre la représentation d'un lieu de différents points de vue textuels. Elle propose une perspective multifocale qui met en évidence la profondeur du lien entre la réalité et sa représentation au point de voir la transformation de la réalité dans sa représentation. Bertrand Westphal, chef de file de la géocritique, affirme que la relation entre l'espace réel et sa représentation littéraire ne peut qu'être « dynamique » et « dialectique ».

La géocritique, en effet, se propose d'étudier non pas seulement une relation unilatérale (espace-littérature), mais une véritable dialectique (espace-littératureespace) qui implique que l'espace se transforme à son tour en fonction du texte qui, antérieurement, l'avait assimilé. Les relations entre littérature et espaces humains ne sont donc pas figées, mais parfaitement dynamiques. L'espace transposé en littérature influe sur la représentation de l'espace dit réel (référentiel), sur cet espace-souche dont il activera certaines virtualités ignorées jusque-là, ou réorientera la lecture ${ }^{21}$.

Selon Westphal le discours fonde l'espace : «[...] Pétersbourg/Dostoievski, Dublin/Joyce, Prague/Kafka, Tanger/Bowles ou encore Lisbonne/Pessoa. Là, espaces humains et littérature sont indissociables ; imaginaire et réalité sont imbriqués ; le référent n'est plus forcément celui que l'on croit. En deux mots, ou en trois, c'est l'écrivain qui est devenu auteur de sa ville. » Dans cette perspective, qui dépasse l'opposition entre réalisme de la représentation et structuralisme textuel, la relation entre lecteur et espace humain, par le biais de la littérature, sera la suivante :

[...] l'espace humain devra cesser de lui paraitre évident; ce qu'il perçoit devra devenir indice d'une compossibilité dont il lui incombera de définir la continuité. Mais, en tout état de cause, si la continuité est symbolisée par une ligne, cette ligne sera une ligne de fuite. Face et dans le temps, l'espace humain est un jardin aux sentiers qui bifurquent à gauche, à droite, en haut, en bas ${ }^{22}$.

\section{Politique de la littérature et connaissance pratique}

Par ailleurs, la possibilité nous est donnée de comparer deux auteurs qui ont récemment contribué à identifier des dimensions spécifiques du récit.

Le premier, Jacques Rancière, par le biais de l'expression "politique de la littérature " met en évidence la capacité intrinsèque de la littérature à définir «le monde que nous habitons : la façon dont il est pour nous visible, et donc ce visible se laisse dire ${ }^{23}[. .]$.$» Il$ introduit sa réflexion en explicitant avant tout les termes clé : politique et littérature.

D'après Rancière, la politique est l'activité humaine qui construit une «sphère d'expérience spécifique» et commune dans laquelle on trouve l'accord sur des coordonnés objectives et subjectives. Il s'agit d'une construction qu'il ne faut pas considérer figée pour toujours mais constamment à rediscuter et redéfinir. Ce procès politique de définition des bornes de l'exprimable et de l'indicible, d'introduction d'objets et de sujets nouveaux, est appelé « partage du sensible ${ }^{24}$ ». Son début a lieu au moment de 
la conquête de la parole par ceux qui, destinés à la production des moyens nécessaires pour vivre, avaient été considérés, par Aristote, des « animaux bruyants ».

D'après Rancière, la littérature, au lieu d'être une catégorie atemporelle incluant l'ensemble des productions artistiques écrites, est « un régime nouveau d'identification de l'art d'écrire ${ }^{25}$ » apparu en Europe au XIX siècle, à savoir " un système de rapports entre des pratiques, des formes de visibilité de ces pratiques, et des modes d'intelligibilité ${ }^{26}$. ». Autrement dit, seulement dans le creuset de la grande transformation de la « sphère d'expérience spécifique » et commune du XIX ${ }^{e}$ siècle on a pu définir le nouveau concept de littérature comme système de relations spécifiques entre l'activité d'écrire, la perception qu'ont les lecteurs de cette activité et la compréhension rationnelle du rapport entre cette pratique et sa réception. En montrant sa capacité politique, la littérature a ainsi constitué une des modalités d'intervention «dans le partage du sensible qui définit le monde que nous habitons ». À partir de cette spécificité il devient possible de penser «la politique de la littérature comme telle» en mettant au jour ses caractéristiques utiles pour la construction de notre « sphère d'expérience spécifique » et commune.

Dans Qu'est-ce que la littérature?, Sartre opposait l'intransitivité poétique à la transitivité littéraire, et repérait la spécificité politique de la littérature dans son emploi des mots comme instruments de communication utiles pour construire un monde commun. Rancière estime au contraire que la littérature ne développe pas une fonction politique par son usage spécifique du langage, mais en intervenant « dans le découpage des objets qui forment un monde commun, des sujets qui le peuplent et des pouvoirs qu'ils ont de le voir, de le nommer et d'agir sur lui ${ }^{27}$. ». Et il illustre cette perspective par des exemples. Il compare deux lectures opposées de l'œuvre de Flaubert, un des écrivains dont l'œuvre semble se décrocher de toute forme de signification politique. La première de ces interprétations, celle des contemporains de Flaubert, voyait dans l'attention au détail (qui mettait le message en arrière plan) une forme extrême d'égalitarisme qui incarnait parfaitement la marée démocratique. De plus, Flaubert mettait en discussion toutes les hiérarchies des sujets représentés. La deuxième de ces lectures, celle de Sartre, voyait au contraire dans le langage purifié, c'est-à-dire pétrifié (soucieux de définir et circonscrire les choses dont il parle et dépourvu de tout message), la forme de sa rébellion aristocratique au monde bourgeois et à son langage prosaïque ouvert à la signification et à la communication. Ce sont deux lectures presque identiques de l'œuvre de Flaubert qui produisent pourtant des interprétations opposées. Comment en comprendre les raisons? Rancière affirme qu'on peut le faire seulement si on considère ces deux interprétations comme faisant partie du même cadre logique de ce dispositif historique, fonctionnel à la reconfiguration du monde, qui s'appelle littérature et qui est né au XIXe siècle.

Dans ce dispositif historique que nous appelons littérature trois pratiques s'entrelacent :

Une pratique d'écriture qui, si d'un côté réalise «la grande loi de l'égalité de tous les sujets et [...] la disponibilité de toutes les expressions ${ }^{28}$ " en dissolvant la «sphère d'expérience" commune précédente faite de hiérarchies sociales et linguistiques aristocratiques, de l'autre côté oppose "à la démocratie de l'écriture ${ }^{29}$ ", qui a permis à tous d'exprimer leur point de vue, de nouveaux rapports entre le mots et les choses permettant la « lecture des lois d'un monde sur le corps des choses banales et des mots sans importance ${ }^{30}$.» théoriques employés plus tard par ceux qui prétendaient démystifier la littérature. C'est 
ce que Rancière appelle la « lecture symptômale ${ }^{31}$ ", c'est-à-dire une lecture en mesure de repérer le symptôme d'un signifié futur dans le renoncement au sens qui se reflète dans l'écriture pétrifiée; une lecture en mesure de dévoiler les lois de la structure sociale cachées dans la fiction narrative. Il ne s'agit pas d'un dévoilement posthume réalisé par la théorie herméneutique mais d'une invention originale de la littérature «[...] qui s'est détournée de la logique des actions prétendument gouvernées par leurs fins rationnelles vers le monde des significations cachées dans l'apparente banalité32. ».

Une pratique herméneutique qui, par rapport à la multiplicité excessive des interprétations qu'elle-même avait provoquées, s'auto-corrige en opposant «à la mutité trop bavarde de la lettre» (conséquence d'une pratique d'écriture qui disperse les signifiés précédents), et «à la parole écrite sur les choses et les corps » (fruit de la « lecture symptômale » qui provoquent le bavardage herméneutique), « la respiration des choses délivrées de l'empire des significations ${ }^{33}$.»

La littérature, en tant que système de relations historiquement déterminées, contribue à renouveler la représentation d'un sens commun à partir du constat de la "pétrification " littéraire qui a eu lieu dans un contexte historique précis, celui du XIX ${ }^{e}$ siècle. Pour comprendre la politique de la littérature il ne suffit pas de la réduire à résultat d'un simple ajustement, toujours partiel et ambigu, entre une forme d'écriture et un contenu politique, mais il faut tenir compte de toutes les tensions contradictoires du procès qui la déterminent. Rancière affirme que :

Le critique ou le sociologue voudront prendre ici leur revanche en faisant de cette contradiction la marque de la vieille illusion qui s'imagine changer la vie quand elle ne fait que l'interpréter. Mais les interprétations sont elles-mêmes des changements réels, quand elles transforment les formes de visibilité d'un monde commun et, avec elles, les capacités que les corps quelconques peuvent y exercer sur un paysage nouveau du commun. [...] Une réflexion sur la politique de la littérature peut alors nous aider à comprendre cette ambiguité et certaines de ses conséquences dans les sciences qui prétendent interpréter le monde comme dans les pratiques qui entendent le transformer ${ }^{34}$.

Dans La connaissance de l'écrivain. Sur la littérature, la vérité et la vie, Jacques Bouveresse souligne la capacité du discours narratif à transmettre une expérience vécue, et suggère ainsi que ce discours est en mesure de transmettre aussi une connaissance pratique de la vérité. Afin d'arriver à cette conclusion Bouveresse se demande si la littérature peut avoir un lien avec la vérité et la connaissance. Il prend en compte et approfondit deux possibilités : la littérature nous donne une connaissance d'essences; la littérature nous donne une connaissance expérimentale.

D'après Bouveresse, l'idée chère aux structuralistes selon laquelle l'unique forme de connaissance serait la connaissance de principes théoriques, est douteuse voire fausse. $\mathrm{Si}$ on se fiait à une conception essentialiste classique, on devrait nécessairement conclure que la littérature n'a aucun rapport avec la connaissance. Récemment, avec le scepticisme postmoderne, une autre conception de la vérité rationnelle s'est fait jour, selon laquelle toutes les disciplines de la réflexion entretiennent la même relation avec la vérité. Bouveresse au contraire affirme que la littérature est en mesure de transmettre une forme de connaissance particulière, à la fois essentielle et immédiate, pouvant atteindre des profondeurs inconnues à la science et à la philosophie. Si la vérité est un récit parmi d'autres récits, il faut reconnaître qu'en tant que telle, elle nous aide à résoudre les problèmes que nous avons avec le monde et avec les autres et que, par voie de conséquence, la littérature est la discipline suprême pour connaitre la vérité. Mais cette 
conception affirme une vérité essentielle sans en détecter les fondements, car elle ne répond pas à la question cruciale : pourquoi la littérature devrait-elle être considérée comme un chemin d'accès particulier et irremplaçable pour accéder à la vérité ? Selon Bouveresse c'est à cette question qu'il faut répondre. Et la réponse qu'il faut donner, qu'il y ait ou non un lien essentiel entre vérité et littérature, est que la connaissance acquise par la littérature est expérimentale.

En citant Putnam, qui soutient: «Si je lis le Voyage au bout de la nuit de Céline, je n'apprends pas que l'amour n'existe pas, que tous les êtres humaines sont odieux et haineux [...]. Ce que j'apprends est à voir le monde comme il a l'air d'être pour quelqu'un qui est sûr que cette hypothèse est correcte ${ }^{35}$.", Bouveresse affirme que ce type de connaissance pratique « a un rapport direct avec la question de savoir comment nous pouvons ou devons $v^{2} v_{r}{ }^{36}$.». Même si ce n'est pas la connaissance de la science théorique, c'est néanmoins un genre de connaissance qui sert à «étendre notre imagination et notre sensibilité morale et améliorer ainsi notre aptitude au raisonnement pratique $^{37}$. ». Les œuvres littéraire en général et le genre romanesque en particulier, sans avoir l'ambition didactique de vouloir corriger un comportement pratique déterminé, apportent une contribution importante à la connaissance (morale) en construisant des milieux contrôlés dans lesquels on fait interagir des personnages afin d'en analyser les comportements face à des choix moraux complexes et conflictuels. De ce point de vue, le roman nous aide dans la compréhension de nous-mêmes en nous permettant « de participer à des expériences de nature extra-morale, que nous n'aurions pas la possibilité [...] de faire dans la vie réelle ${ }^{38} "$, mais nous montre aussi la limite de la philosophie morale et de la morale même. S'opposant au moralisme ou kantisme de la contemporanéité, c'est-à-dire à la recherche, en matière d'éthique, de l'inconditionnalité et de l'univocité, les romanciers (attirés par l'ambiguïté et par l'indécision) ne veulent pas former des jugements clairs et distincts mais veulent tout simplement contempler des situations équivoques du point de vue éthique en les explorant jusqu'au bout par le moyen de leur imagination morale. Selon Bouveresse, qui accueille la réflexion de Martha Nussbaum ${ }^{39}$, c'est dans cela, et non dans un prétendu lien essentiel entre politique et littérature (comme l'affirmait Rancière), qu'on pourrait repérer la politique de la littérature, sa dimension publique. Le roman, en nous proposant des possibilités auxquelles nous ne pensons pas normalement, nous aide à prendre position dans la sphère privée, autant que dans la sphère publique. La spécificité du roman, en tant que "paradigme d'un style de raisonnement éthique qui est spécifique, par rapport au contexte, sans être relativiste», est que «nous arrivons à des prescriptions concrète universalisables en faisant porter une idée générale de l'épanouissement humain sur une situation concrète dans laquelle nous sommes invités à entrer par l'imagination ${ }^{40}$.». L'importance du genre romanesque, du point de vue éthique, consiste donc dans ce « vaet-vient spécifique entre le général et le concret ${ }^{41} »$ qui, en nous ouvrant des possibilités que nous ne serions pas capables d'expérimenter, nous permet d'approfondir notre connaissance morale, au point de mettre en discussion, et potentiellement de renverser, le moralisme dominant.

\section{Vérité de la paralittérature}

41 On vient de voir comment, pour Bouveresse, le roman, en racontant l'existence des êtres humains qui ont affaire au monde, ne concerne pas uniquement le problème de la 
connaissance théorique mais, en mettant en relation la pensée et l'action, devient l'outil privilégié d'une réflexion éthique autonome.

Or: est-il toujours possible de supposer cette autonomie? Est-il possible d'évaluer la contribution de vérité qu'apporte un produit culturel sans se référer à la production esthétique contemporaine, sans situer ce produit dans une société fondée sur la production industrielle des marchandises?

En fait, la forme narrative romanesque ne peut être évaluée, du point de vue de sa capacité à transmettre quelque chose sur le monde, sans se référer à la forme sociale globale qui la détermine et qu'elle contribue à déterminer. On sait que, dans un monde dominé par la logique de la valorisation commerciale du capital engagé dans la production des marchandises, l'art devient une production esthétique ou un système $\mathrm{d}^{\prime}$ ' industrie culturelle ${ }^{42}$.». Comme la théorie marxienne du fétichisme des marchandises nous l'a appris, cela signifie que la vérité de l'art, comme la vérité de toute autre marchandise, réside dans le fait qu'elle est vendable. La valeur d'échange de la «marchandise-art» se détache de la particularité concrète de l'objet échangé, de sa valeur d'usage. Elle s'émancipe de la vérité de son contenu et de son contenu moral, pour se soumettre à la vérité de sa forme marchande. La marchandise est obligée de réaliser à chaque instant et en tout lieu sa valeur d'échange. Pour cela, il faut que le produit artistique soit universellement reconnaissable et il faut qu'il soit un produit sériel. Voilà qui est en contradiction avec la nature même du produit esthétique, par définition unique et original.

Pour en revenir à la littérature, comment peut-on conjuguer l'originalité d'un objet littéraire avec la nécessité sérielle de la valorisation du capital investi dans sa production? D'après Benjamin, en faisant apparaitre le nouveau dans le toujours-égal et le toujours-égal dans le nouveau. En définitive, le nouveau artistique, pour exister, doit confirmer le toujours-égal de la marchandise. Pour les objets littéraires cette condition générale ne peut se réaliser qu'à travers une formalisation extrême des structures narratives afin de minimiser le risque, toujours présent, de ne pas atteindre le but commercial, la valorisation à travers la vente. Dans le roman contemporain, surtout dans le plus commercial, la supériorité des constantes narratives et idéologiques par rapport aux particularités mimétiques, fait partie non seulement de la sérialité productive, de l'éternelle représentation du toujours-égal, mais constitue aussi l'une des principales raisons de son succès auprès du public. C'est précisément la répétitivité du déroulement, des personnages, du contexte sociologique, qui rend, par exemple, les romans policiers si "familiers ", si « rassurants ", et donc si populaires. Cette fixité formelle n'entraîne pas cependant un état statique pur et simple. A l'uniformité formelle s'ajoutent chaque fois des éléments de nouveauté, tels que le moment conflictuel, nécessairement original, du crime.

La soi-disant « paralittérature » ou production narrative low brow, nous met constamment devant le paradoxe du retour du couple dialectique "répétition/innovation ». En tant qu'élément perturbateur de l'ordre initial, l'imprévu, inconnu et déstructurant, est en même temps l'élément connu et structurant, inclus dans le projet de simplification totalitaire de l' «industrie culturelle» et fait partie de l'occultation allégorique du rapport signifiant/signifié typique de la production esthétique moderne. Pour toutes ces raisons, on peut estimer que la production romanesque qui se fonde plus que toute autre sur la répétition et la sérialisation des éléments familiers par rapport à des éléments prétendument originaux, c'est-à-dire la paralittérature, offre une meilleure possibilité 
d'interprétation de la réalité sociale contemporaine, de plus en plus marquée par le processus de valorisation capitaliste, ce qui n'est pas le cas de la production littéraire high brow.

\section{8. Épiques et nouveaux engagements}

En guise de conclusion, on souhaite mentionner les thèses d'un groupe d'écrivains italiens Wu Ming ${ }^{43}$. En mettant en œuvre ce qu'ils appellent New Italian Epic, ils ont développé le caractère "inquiétant ", à la fois étranger et familier, nouveau et toujours-égal, d'un corpus de narration romanesque qui, d'après eux, seulement par l'altération et par la déformation de la réalité, a récupéré la fonction éthique et réaliste de la transmission d'un patrimoine commun de croyances, symboles et allégories.

Selon ce collectif d'écrivains, l'époque comprise entre 1993 et 2008 est caractérisée, en Italie, par l'apparition d'une "nébuleuse d'œuvres ${ }^{44}$ », ou "nébuleuse narrative ${ }^{45}$ », qui révèle une nouvelle conscience éthique et le retour à « un fort sens de responsabilitét6 " de la part d'écrivains dégoûtés par le cynisme postmoderne.

Des œuvres comme Dies Irae de Giuseppe Genna, 54 de Wu Ming, Romanzo criminale de Giancarlo de Cataldo, Black Flag de Valerio Evangelisti, sont "New» parce qu'en employant des éléments stylistiques et thématiques qui ne rompent pas avec la tradition, elles déclenchent des réactions qui font jaillir le nouveau de l'existant. Dans ces œuvres le monde est perçu de points de vues insolites (un sorcier pendant la Révolution industrielle, un téléviseur américain dans l'Italie des années Cinquante): il perd la masque familier qu'on connaît et devient paradoxalement mieux interprétable. Des procédés narratifs typiques de la paralittérature (anticipations, coups de théâtre, etc.) sont mis au service d'une remarquable complexité de la narration. Des articulations historiques décisives sont saisies à travers la construction de réalités potentielles et alternatives. Des expériences sont dissimulées dans des registres typiques des genres narratifs "bas». Toutes ces caractéristiques, présentes en mesure plus ou moins importante dans les travaux cités, participent du caractère "inquiétant» de cette littérature. Ces œuvres confirment l'impossibilité d'atteindre une vérité ultime à travers la littérature, mais elles démontrent, selon une perspective benjamininienne bien connue de Wu Ming, qu'existe la possibilité, tandis que nous marchons, le visage tourné vers le passé, de chercher, grâce à la littérature, « à voir l'avenir ${ }^{47}$. ».

49 Ensuite, ces textes sont «Italian», et il ne peut pas en être autrement à cause de l'anomalie persistante que représente l'Italie toujours déchirée et en guerre avec ellemême, dans le passé, pendant la Guerre froide par exemple, comme aujourd'hui. Ce conflit, en un premier temps, a vu un retour aux genres paralittéraires, les seuls qui étaient en mesure de développer une enquête réaliste du tragique social moderne. Plus tard quand la " poussée propulsive ${ }^{48}$ » des genres paralittéraires a commencé à s'épuiser, est intervenu un dépassement de la forme romanesque qui a abouti à ce que Wu Ming définit : «objet narratif non-identifié ${ }^{49}$. ». Les UNO (Unidentified Narrative Object) «sont intéressants et innovants pour leur façon de compléter les caractéristiques de la nébuleuse narrative et non parce qu'ils prennent le chemin du réalisme en mélangeant événements et fictions, histoires et témoignages ${ }^{50}$.» Exemple exquis d'UNO : Gomorra de Roberto Saviano. 
Et enfin, ces œuvres sont «Epic » parce que, à la différence des œuvres postmodernes elles sont faites de "ardeur civil, colère, douleur pour la mort du père, amour fou et empathie avec qui souffre ${ }^{51}$." L'épique, dans l'interprétation de Wu Ming, "est une hyperbole qui produit une friction - ou tout à fait une collision ouverte - entre le familier et l'étranger ", " elle a à voir avec le Unheimliche, l'attraction exercée par le non-familier ${ }^{52}$ .». Elle récupère l'«éthique de la narration », la "confiance dans le mot et dans la possibilité de le réactiver, de le recharger de signification ${ }^{53}$ ", après le désenchantement et la désillusion typiques de la conscience postmoderne de la fin des grandes narrations, qui se sont épuisées dans des stériles formalismes. L'épique rachète «la forme promenade ", l'attitude subversive du flâneur qui ne s'arrête pas aux apparences de l'identité, mais trouve l'identité dans les apparences.

Peut-on parler, comme cela a été fait, de néo-néo-réalisme pour la New Italian Epic ? Selon Wu Ming il s'agit d'un faux problème. Si le réalisme s'occupe du côté dénotatif du langage en choisissant de rester proche "du (tangible, très matériel) "compromis perceptif" nommé "réalité54" ", l'épique en revanche nait du passage à travers tous les signifiés : elle renvoie sans fin à la dimension connotative du récit et du langage. Même si ces deux dimensions sont profondément différentes, elles ne s'excluent pas réciproquement. $\mathrm{Au}$ contraire, elles coexistent souvent dans la même œuvre et définissent les contours de l'UNO. «Dans la littérature, n'importe quelle recherche du "réalisme", n'importe quelle tentative de représentation "objective", doit tenir compte de tout cela : notre pensée est "figurée", les connotations prolifèrent, l'allégorie nous échappe de tous les côtes [...], l'épique est un débouché naturel ${ }^{55}$ [...].»

\section{Conclusions}

En guise de bilan, on souligne que la mimésis narrative, en reconfirmant son altérité par rapport aux formalisations philosophiques ou scientifiques, contribue de nouveau, au lendemain de l'ivresse postmoderniste, à la création d'un point de vue capable de transmettre/recevoir, édifier/démolir, la connaissance de vérité sur la vie et sur le monde. Une connaissance qui chaque fois, selon les différents interprètes, peut être: politique, morale ou épique. En ce qui concerne la littérature italienne du passé récent, le roman, dans sa forme d'UNO, reste le seul chemin possible pour reconstruire une totalité toujours partielle de significations. Le roman rebâtit le monde par un langage connotatif. Un monde divisé dont désormais, dans cette société sans passé, on a perdu les coordonnées. Un monde presque impossible à décrire par un langage dénotatif, qui serait objectivement complice de sa progressive perte de sens. L'écriture italienne contemporaine, en restituant son mystère à ce " hiéroglyphique social » qu'est la formemarchandise, en démystifie paradoxalement l'incompréhensibilité et l'absurdité. En reconstruisant l'illogisme du réel, elle met en doute sa nature mythique et indiscutable, en rétablissant la dimension épique de l'action, elle démasque le vulgaire prosaïsme de l'inertie. 


\section{BIBLIOGRAPHIE}

T. Adorno et M. Horkheimer, La Dialectique de la Raison, traduction de Eliane Kaufholz, Gallimard, 1974.

H. de Balzac, Avant-Propos de La Comédie humaine, Gallimard, Paris, 1976.

W. Benjamin, Charles Baudelaire : un poète lyrique à l'apogée du capitalisme, traduction de Jean Lacoste, Payot, Paris,1974.

W. Benjamin, Thèses sur la Philosophie de l'Histoire, Essais 2, traduction de Maurice de Gandillac, Denoêl-Gauthier, Paris, 1935-1940.

G. Bonino, Universali/particolari, Il Mulino, Bologna, 2008.

J. Bouveresse, La connaissance de l'écrivain. Sur la littérature, la vérité et la vie, Agone, Marseille, 2008.

S. Givone, Il bibliotecario di Leibniz, Einaudi, Torino, 2005.

L. Geymonat, Storia del pensiero filosofico e scientifico, Garzanti, Milano, 1970.

G. Lukàcs, Problemy teorii romana, dans Literaturnyj kritik nn. 2 et 3, 1935, trad. it. Vittorio Strada, Problemi di teoria del romanzo, Einaudi, Torino, 1976.

G. Mazzoni, Mimesi, narrativa, romanzo, dans Moderna. Semestrale di teoria della letteratura, VII, 2.2005, Istituti Editoriali e Poligrafici Internazionali, Pisa-Roma.

J. Rancière, Politique de la littérature, Galilée, Paris, 2007.

B. Westphal, La géocritique mode d'emploi, PULIM, Limoges, 2000.

Wu Ming, New Italian Epic. Letteratura, sguardo obliquo, ritorno al futuro, Einaudi, Torino, 2009.

Wu Ming 1 et Wu Ming 2, Il N.I.E. è morto, www.carmillaonline.com, 16/06/2009.

\section{NOTES}

1. G. Mazzoni, Mimesi, narrativa, romanzo, dans Moderna. Semestrale di teoria della letteratura, VII, 2.2005, Istituti Editoriali e Poligrafici Internazionali, Pisa-Roma, p. 21. C'est nous qui traduisons.

2. L. Geymonat, Storia del pensiero filosofico e scientifico, Garzanti, Milano, 1970. C'est nous qui traduisons.

3. G. Bonino, Universali/particolari, Il Mulino, Bologna, 2008, p. 15. C'est nous qui traduisons.

4. H. de Balzac, Avant-propos de La Comédie humaine, Gallimard, Paris, 1976, v. 1, p. 11.

5. S. Givone, Il bibliotecario di Leibniz, Einaudi, Torino, 2005, pp. 6-7. C'est nous qui traduisons.

6. S. Givone, Idem, pp. 10-11. C'est nous qui traduisons.

7. S. Givone, Idem, p. 13. C'est nous qui traduisons.

8. S. Givone, Idem, p. 11. C'est nous qui traduisons.

9. S. Givone, Idem, p. 37. C'est nous qui traduisons.

10. S. Givone, Idem, p. 16. C'est nous qui traduisons.

11. La notion pragmatique de «jeu linguistique» développée par Wittgenstein, dans ses Philosophische Untersuchungen, entend délimiter l'usage quotidien des mots ou de la pensée. Cité par G. Mazzoni, Mimesi, narrativa, romanzo, dans Idem, p. 22. C'est nous qui traduisons. 
12. S. Givone, Idem, p. 57. C'est nous qui traduisons.

13. S. Givone, Idem, p. 58. C'est nous qui traduisons.

14. G. Lukàcs, Problemy teorii romana, dans Literaturnyj kritik nn. 2 et 3,1935, trad. it. Vittorio Strada, Problemi di teoria del romanzo, Einaudi, Torino, 1976. C'est nous qui traduisons.

15. G. Lukàcs, Il romanzo come epopea borghese, dans Idem. C'est nous qui traduisons.

16. G. Lukàcs, Ibidem. C'est nous qui traduisons.

17. S. Cavell, The Claim of Reason: Wittgenstein, Skepticism, Morality, and Tragedy, Oxford University Press, Oxford, 1979, dans S. Givone, Idem, p. 119. C'est nous qui traduisons.

18. Le concept de "formation discursive", introduit par Foucault dans L'Archéologie du savoir (Paris, Gallimard, 1969) entend mettre en lumière l'institution de hiérarchies de textes et de savoirs qui, sédimentés peu à peu au cours de l'histoire, se coagulent en structures rigides et codifiées.

19. G. Mazzoni, Mimesi, narrativa, romanzo, dans Idem, p. 46. C'est nous qui traduisons.

20. G. Mazzoni, Mimesi, narrativa, romanzo, dans Idem, p. 53. C'est nous qui traduisons.

21. B. Westphal, La géocritique mode d'emploi, PULIM, Limoges, 2000, p. 22, et aussi http:// www.vox-poetica.org/sflgc/biblio/gcr.htm

22. B. Westphal, Idem, p. 28.

23. J. Rancière, Politique de la littérature, Galilée, Paris, 2007, p. 15.

24. J. Rancière, Idem, p. 12.

25. J. Rancière, Idem, p. 15.

26. J. Rancière, Ibidem.

27. J. Rancière, Ibidem.

28. J. Rancière, Idem, p. 30.

29. J. Rancière, Ibidem.

30. J. Rancière, Idem, p. 31.

31. J. Rancière, Idem, p. 32.

32. J. Rancière, Idem, p. 31.

33. J. Rancière, Idem, p. 35.

34. J. Rancière, Idem, pp. 39-40.

35. H. Putnam, Literature, Scienceand Reflection, in Meaning and the Moral Sciences, Rouledge \& Kegan Paul, Londres-Henley-Boston, 1978, dans J. Bouveresse, La connaissance de l'écrivain. Sur la littérature, la vérité et la vie, Agone, Marseille, 2008, p. 59.

36. J. Bouveresse, Idem, p. 63.

37. J. Bouveresse, Idem, p. 63-64.

38. J. Bouveresse, Idem, p. 139.

39. M. Nussbaum, Poetic Justice. The Literary Imagination and Public Life, Beacon Press, Boston, 1995, dans J. Bouveresse, Idem.

40. M. Nussbaum, Idem, dans J. Bouveresse, Idem, p. 161.

41. J. Bouveresse, Idem, p. 160.

42. T. Adorno et M. Horkheimer, La Dialectique de la Raison, trad. Eliane Kaufholz, Gallimard, 1974.

43. Wu Ming signifie « sans nom » en chinois.

44. Wu Ming 1 et Wu Ming 2, Il N.I.E. è morto, interview donné au site www.carmillaonline.com, 16/06/2009. C'est nous qui traduisons.

45. Wu Ming, New Italian Epic. Letteratura, sguardo obliquo, ritorno al futuro, Einaudi, Torino, 2009, p. 10. C'est nous qui traduisons.

46. Wu Ming, Idem, p. IX. C'est nous qui traduisons.

47. Wu Ming, interview dans Idem. C'est nous qui traduisons.

48. Wu Ming, Idem, p. 20. C'est nous qui traduisons.

49. Wu Ming, Idem, p. 41. C'est nous qui traduisons.

50. Wu Ming, interview dans Idem. C'est nous qui traduisons. 
51. Wu Ming, Idem, p. 25. C'est nous qui traduisons.

52. Wu Ming, Idem, p. 72. C'est nous qui traduisons.

53. Wu Ming, Idem, p. 24. C'est nous qui traduisons.

54. Wu Ming, Idem, p. 68. C'est nous qui traduisons.

55. Wu Ming, Idem, p. 71. C'est nous qui traduisons.

\section{RÉSUMÉS}

La mimésis narrative, en reconfirmant son altérité par rapport aux formalisations philosophiques ou scientifiques, contribue de nouveau, au lendemain de l'ivresse postmoderniste, à la création d'un point de vue capable de transmettre/recevoir, édifier/démolir, la connaissance de vérité sur la vie et sur le monde. Une connaissance qui chaque fois, selon les différents interprètes, peut être : politique, morale ou épique. En ce qui concerne la littérature italienne du passé récent, le roman, dans sa forme d'UNO (Unidentified Narrative Object), reste le seul chemin possible pour reconstruire une totalité toujours partielle de significations. L'écriture italienne contemporaine, en restituant son mystère à ce "hiéroglyphique social " qu'est la forme-marchandise, en démystifie paradoxalement l'incompréhensibilité et l'absurdité.

La mimésis narrativa, proprio riconfermando la sua essenziale alterità rispetto alle formalizzazioni sistematiche di natura filosofica o scientifica, contribuisce di nuovo, all'indomani della sbornia postmoderna, alla costruzione di un punto di vista in grado di trasmettere/ricevere, costruire/demolire, la conoscenza di un qualche genere di verità sulla vita e sul mondo. Una conoscenza che, secondo i differenti interpreti, può assumere caratteristiche politiche, morali o epiche. Per quanto riguarda la narrativa italiana del recente passato, il romanzo, nella sua attuale forma UNO (Unidentified Narrative Object), rimane l'unica strada possibile, nel mondo scisso dell'esperienza borghese, per ricostruire una totalità sempre parziale di significato. La narrativa italiana contemporanea, restituendo enigmaticità al « geroglifico sociale » della forma merce, ne demistifica paradossalmente l'incomprensibilità e l'assurdità.

\section{INDEX}

Index chronologique : XXe siècle, XXIe siècle

Mots-clés : connaissance pratique, épique, épistème, espace, marchandises, mimésis, œuvre d'art, paralittérature, politique de la littérature, roman, temps, transmission, UNO (Unidentified Narrative Object), vérité

Index géographique : Italie

\section{AUTEUR}

\section{FAUSTO BONI}

Doctorant à l'Université de Nice-Sophia Antipolis, CIRCPLES 